\title{
Variations sur la réussite
}

\section{HRONIQUE • Formation des maitres}

La question de la réussite et de l'échec à l'école constitue sans doute un enjeu majeur des démocraties occidentales. Peu de questions, en effet, engagent autant l'avenir d'une société, ses idéaux de justice et d'équité, son projet social et politique. Cette question nous concerne évidemment comme parents et citoyens et interpelle aussi les formateurs d'enseignants, les enseignants et les futurs enseignants. Sous la forme de variations sur la réussite, je me propose, dans ce texte, de contribuer à la clarification des notions souvent confondues de réussite scolaire et de réussite éducative. Je voudrais aussi montrer que ces notions sont indissociables d'une réflexion sur ce que les Anciens appelaient la vie bonne, et que nous nommons aujourd'hui la vie réussie.

L'histoire de l'éducation et des institutions éducatives est très utile pour examiner la question de la réussite scolaire. Utile, car elle nous enseigne que la réussite et l'échec à l'école sont des constructions sociales historiquement situées (Kahn, 2011). En d'autres termes, des notions relatives, à un double titre : d'abord, parce qu'elles varient dans le temps et selon les traditions éducatives et culturelles; ensuite, parce qu'il s'agit toujours de comparer ou de mettre en relation des individus par rapport à une norme elle-même relative. De ce point de vue, ce qui est considéré comme un échec ou une réussite à une époque ou dans un certain contexte éducatif et culturel peut ne pas l'être ou n'avoir aucun sens à une autre époque ou dans un autre contexte; de la même manière, ce qui est un échec pour un élève peut être une réussite pour un autre. Cette simple mise en perspective n'invalide pas la notion de réussite scolaire telle que nous la 
définissons habituellement dans les discussions actuelles, elle a simplement pour effet d'en montrer le caractère contingent et relatif, et donc possiblement mouvant, changeant, évolutif. Elle montre aussi que la norme définie par l'institution produit des effets sur notre conception de la réussite et de l'échec scolaires (Kahn, 2011). En poussant le raisonnement aussi loin que possible, on pourrait dire que c'est la norme qui produit la réussite ou l'échec, pas l'élève. En d'autres termes, et pour le dire rapidement, l'échec et la réussite scolaires ne sont jamais des qualités intrinsèques à l'élève.

Ce qu'on appelle aujourd'hui la réussite scolaire, il faut le rappeler, a bien le sens d'une performance scolaire mesurée par des épreuves, des évaluations, des tests standardisés qui attestent qu'on a fait les apprentissages prévus par l'institution, c'est-à-dire les apprentissages planifiés d'une manière uniforme pour tous les élèves d'un même degré, selon une progression précise et un rythme défini. Inversement, s'il y a échec, c'est que le seuil minimal de la performance n'a pas été atteint. Quand le ministère de l'Éducation, du Loisir et du Sport nous dit qu'il faut « hausser le taux de diplomation ou de qualification à $80 \%$ chez les élèves de moins de 20 ans d'ici 2020 »(MELS, 2009, p. 7), c'est bien de cela dont il s'agit, de réussite scolaire au sens où je l'ai définie. Des progrès ont été faits à cet égard et nous connaissons mieux aujourd'hui les conditions favorables à la réussite à l'école. J'en rappelle quelques-unes brièvement. Premièrement, une volonté politique claire, ferme, manifeste, qui met les élèves, les enseignants, l'école au cœur de son projet politique; deuxièmement, une collaboration et une concertation nettement affirmées au sein même de l'école, entre le personnel et la direction, mais aussi entre l'école et les parents, entre l'école et son milieu; troisièmement, une fine et patiente analyse des besoins de chaque enfant pour améliorer la première scolarisation; quatrièmement, une école ouverte, accueillante, qui diversifie son offre de formation sans discriminer et qui prévoit des mécanismes d'assouplissement pour ne pas enfermer, bloquer ou déterminer trop tôt l'avenir de nos jeunes; cinquièmement, des pratiques pédagogiques rigoureuses et structurées; sixièmement, des enseignants enthousiastes et compétents et des parents qui valorisent l'éducation. Je m'arrête ici, d'autres conditions existent sans doute, qui sont présentes à des degrés variables. Mais j'ajouterais simplement que dans une société démocratique, qui défend un idéal d'équité, d'égalité et de justice, et dans une société qui fait de l'école la voie de passage obligée pour s'intégrer à la vie sociale et professionnelle, l'école n'est pas là pour nuire à cette éventuelle intégration, pour entraver, flétrir, hypothéquer l'avenir de nos enfants, mais pour contribuer à la réalisation de soi des êtres humains. Je pense que l'école fait partie des conditions qu'une société démocratique doit mettre en place et garantir pour assurer à ses membres une forme de vie réussie (Honneth, 2006). Dans cette perspective, ce n'est pas $80 \%$ des élèves qu'il faut amener à la diplomation ou à la qualification, mais $100 \%$ des élèves. C'est bien là un enjeu éthique, social et politique de première importance.

J'ai parlé de réussite scolaire, j’en viens à ce que j’ai appelé la réussite éducative. Puisant dans ma propre expérience scolaire, me reviennent à l'esprit trois figures, trois noms ${ }^{1}$, trois ordres d'enseignement : Lucille Beaulieu en $5^{\mathrm{e}}$ et $6^{\mathrm{e}}$ années, Claude Légaré en classe de chimie de $4^{\mathrm{e}}$ secondaire, Pierre-Claude Auger en classe de philosophie, première année de cégep. Qu'avaient-ils en commun? Assurément trois choses indispensables : la passion de l'enseignement et de l'apprentissage, la passion des élèves, concrets, en chair et en os, devant eux, avec eux, et la passion du savoir. Grâce à eux, j’ai compris que l'expérience scolaire pouvait viser autre chose que la seule réussite scolaire et constituer une véritable expérience éducative, celle qui fait événement dans sa vie d'élève, qui transforme de manière durable et qui permet de se découvrir, de connaître un peu plus ses forces, ses qualités, ses ambitions. J'ai appris au 
contact de ces enseignants que l'école pouvait être liée à un concept dense d'éducation, de transmission et de culture. J'ai compris plus tard le vrai sens du mot école, scholè en grec, qui veut dire loisir, non pas divertissement, mais l'attention soutenue à la chose apprise et enseignée, le temps consacré aux études, le temps pour explorer et découvrir ce que d'autres hommes ont fait, pensé, créé, le temps pour s'approprier une partie de l'expérience humaine considérée comme culture, le temps pour se découvrir et se connaître soi-même (Simard, 2004). La réussite éducative ainsi comprise implique aussi un enjeu identitaire et culturel. Qu'est-ce, alors, qu'une éducation réussie? M'inspirant de ces trois formidables enseignants, je dirais ce qu'Olivier Reboul disait si bien dans son ouvrage La Philosophie de l'éducation: une éducation réussie c'est une éducation "inachevée ", celle qui «donne [à l'élève] les moyens et le désir de la poursuivre, d'en faire une auto-éducation » (1989, p. 121), de s'éduquer sa vie durant, car on ne finit jamais de devenir un homme.

Parlant de cette éducation réussie, celle qui donne « les moyens et le désir » de prendre en charge sa propre éducation, de s'éduquer soi-même, j’en viens à la dernière variation : "réussir sa vie ». Qu'estce que réussir sa vie? Mieux encore, qu'est-ce qu'une vie réussie, question qui renvoie forcément à une représentation éthique de la vie bonne? Ici encore, plusieurs réponses sont possibles. L'histoire en regorge, des Grecs jusqu'à nous, pour considérer la seule tradition occidentale. Mais deux choses cependant paraissent pour le moins évidentes de nos jours. La première est qu'il est probablement pour chacun beaucoup plus difficile aujourd'hui qu'autrefois d'apporter une réponse claire à cette question. Et personne ne pourrait prédéterminer pour un autre le choix qu'il revient à chacun de faire. Nous vivons dans des sociétés pluralistes, plus complexes et nettement plus exigeantes sur le plan social, culturel, politique, cognitif, identitaire. Comment s'étonner, dans un tel contexte, que nos jeunes aient parfois beaucoup de mal à s'orienter? De nos jours, les choix possibles sont plus nombreux qu'autrefois, une grande variété de projets de vie s'offre à chacun. Mais l'abondance a aussi ses revers : l'indécision, l'anxiété, l'allongement des études en sont les symptômes les plus connus. Au fil de ma carrière, très souvent j'ai vu des jeunes incertains de leur choix, inquiets devant la diversité qui s'offrent à eux, et toujours je reprenais les mêmes mots pour les rassurer: prenez votre temps en vous rappelant que cette étape, marquée par le doute, l'hésitation et l'ambivalence, est sans doute nécessaire, cruciale, voire salutaire dans la mesure où elle pourrait vous conduire à choisir un projet d'études mieux accordé à ce que vous êtes et à votre projet de vie. Quant à la deuxième évidence, celle qui est au cœur de la modernité et qui explique en partie la première, elle est liée au fait que nous vivons dans des sociétés qui donnent à chacun la possibilité de choisir sa propre vie, de vivre en fonction de sa vision du monde et de ses valeurs, de choisir ses engagements. Et cette conquête, personne ne voudrait la remettre en question aujourd'hui. Alors se pose à chacun la grande question que je rappelle : qu'est-ce que réussir sa vie? Qu'est-ce qu'une vie réussie? Je pense que tout projet d'éducation et de formation culmine dans cette ultime question, tout projet d'études culmine dans un projet de vie. Alors, qu'est-ce qu'une vie réussie? Je risque une réponse : c'est une vie que j'ai librement choisie, celle dans laquelle je me reconnais, celle dans laquelle je me réalise au meilleur de moi-même, celle que je mène dans le respect des autres et des institutions. L'éducation est le fondement de cette liberté. 


\section{Références}

Honneth, A. (2006). La société du mépris : vers une nouvelle Théorie critique. Paris : La Découverte.

Kahn, S. (2011). La relativité historique de la réussite et de l'échec scolaires. Éducation et francophonie, 39(1), 54-66. http://dx.doi.org/10.7202/1004329ar

Ministère de l'Éducation, du Loisir et du Sport (MELS). (2009). Tous ensemble pour la réussite - L'école j’y tiens! Repéré à http://www.education.gouv.qc.ca/fileadmin/site web/documents/dpse/formation jeunes/LEcoleJyTiens TousEnsemblePourLaReussiteScolaire.pdf

Reboul, O. (1989). La Philosophie de l'éducation. Paris : Presses universitaires de France.

Simard, D. (2004). Éducation et herméneutique. Contribution à une pédagogie de la culture. Québec, QC : Presses de l'Université Laval.

\section{Note}

1 Ces noms sont fictifs.

\section{Pour citer cet article}

Simard, D. (2016). Variations sur la réussite. Formation et profession, 24(1), 77-80. http://dx.doi.org/10.18162/fp.2016.a89 